\title{
Primera sistematización de las características estilísticas de la alfarería fina del sitio Soria 2 (Valle de Yocavil, Noroeste argentino)
}

\author{
Romina C. Spano
}

RMA

Arqueología
CONICET, Museo Etnográfico "Juan B. Ambrosetti", Facultad de Filosofía y Letras, Universidad de Buenos Aires. E-mail: romina.spano@gmail.com

\begin{abstract}
Resumen
Se presenta una primera sistematización de las características de la alfarería temprana del sitio Soria 2 (valle de Yocavil, Noroeste Argentino), centrando el análisis en ejemplares pertenecientes al denominado conjunto fino. Se apunta a la caracterización de una muestra del abundante material cerámico hallado en un contexto primario, para el cual se cuenta con un fechado de inicios de la Era Cristiana. El material es clasificado recurriendo a la categoría estilo, entendiendo a la misma como la integración de aspectos morfológicos, tecnológicos y decorativos, que convergen en los "modos de hacer" vigentes durante la ocupación del sitio. Se detallan las variables analíticas puestas en juego: forma, técnica de manufactura, pasta, cocción, tratamiento de la superficie y decoración. La conjunción de dichas variables es la base para proponer modalidades estilísticas. Adicionalmente, se refiere brevemente a las prácticas en las cuales las vasijas estuvieron involucradas, tomando en cuenta los contextos de hallazgo (doméstico y funerario). El análisis sugiere que algunos ejemplares de la muestra estudiada exhiben afinidades con espacios circundantes.
\end{abstract}

Palabras clave: alfarería; modalidades estilísticas; contexto primario; Formativo; valle de Yocavil.

First systematization of stylistic characters of fine pottery from Soria 2 site (Yocavil valley, Northwestern Argentina)

\begin{abstract}
In this paper we present a first systematization of the features of early pottery found at the site Soria 2 (Yocavil Valley, Northwestern Argentina), focusing the analysis on the specimens belonging to the so-called fine pottery group. We aim at the characterization of a sample of the abundant ceramic material found in primary context for which there is a radiocarbon date from the beginning of the Christian era. The material is classified using the style category, considered here as the integration of morphological, technological and decorative aspects, which converge in the current "ways of doing" at those times of the site occupation. The analytical variables used are detailed: shape, manufacturing technique, paste, firing, surface treatment and decoration. The combination of these variables is the basis for the proposed stylistic modes. Additionally, we briefly discuss the practices in which the vessels were involved, taking into account the recovery contexts (domestic and funeral areas). The analysis suggests that some vessels of the ensemble exhibit stylistic affinities with others from surrounding spaces.
\end{abstract}

Keywords: pottery; stylistics modes; primary context; Formative Period; Yocavil Valley.

Se presenta una primera sistematización de las características de una muestra del abundante material cerámico hallado en el sitio Soria 2 (Andalhuala-Banda, sur del valle de Yocavil, Noroeste Argentino -NOA-), un contexto primario interpretado como área de vivienda. Se trata de uno de los pocos contextos de tipo doméstico conocidos hasta el momento correspondiente a los inicios del primer milenio, en el sector meridional de Yocavil, que han podido ser fechados (Palamarczuk et al. 2007; para una síntesis, véase Scattolin 2007). En virtud de este hecho, consideramos que el corpus de información generado a partir del estudio de un espacio doméstico, y de alfarería hallada en posición primaria, puede resultar de interés para la conformación del conocimiento acerca de la cerámica temprana vallista en particular, y de las sociedades agropastoriles en general.

Nuestra propuesta tiene como objetivo efectuar una caracterización estilística del material; a partir de la consideración de diversos atributos, efectuaremos un acercamiento a los posibles modos de hacer objetos cerámicos que estuvieron vigentes entre los antiguos ocupantes del sitio estudiado. Si bien esta contribución quizás podría considerarse como de índole eminentemente descriptiva, entendemos que es preciso dar a conocer los pasos que se siguieron en la construcción de las categorías clasificatorias empleadas, como una primera instancia necesaria en el proceso de indagación acerca de 
la comunidad que produjo y usó la alfarería en cuestión. De este modo, se proporciona información de base, abordada según las distintas variables analíticas puestas en juego.

Asimismo, se alude a información contextual acerca de las situaciones en las cuales las vasijas estuvieron involucradas. Las características del conjunto se consideran, a su vez, a la luz del universo alfarero conocido hasta el momento para el Valle y sus alrededores.

\section{Instrumentos teórico-metodológicos para el análisis}

El estudio del conjunto alfarero fue encarado en términos de análisis estilístico. Es necesario explicitar algunas ideas que tomamos como punto de partida en relación al concepto de estilo que deseamos emplear, ya que se trata de una categoría polisémica que en los estudios de materiales arqueológicos ha sido entendida de maneras diversas, según el momento histórico de la disciplina y las corrientes teórico-metodológicas en vigencia (Conkey y Hastorf 1990; Hegmon 1992; Rice 1987). Nos interesa retomar la propuesta de Hodder (1990) acerca del estilo como modo de hacer, es decir, de elaborar los artefactos de acuerdo a principios y prácticas propias de una sociedad, a lo largo del tiempo; esto es, que la producción de objetos materializa los modos en los que los sujetos y los grupos sociales ven y ordenan la realidad (Earle 1990). En un mismo sentido, Levine (1957) sostiene que el estilo no sería independiente del contenido, porque expresa el mundo perceptual del sujeto, condicionado a la vez por su propia historia de vida y por el grupo humano al cual pertenece. De este modo, el estilo implicaría una relación dialéctica: por un lado, está condicionado por la propia sociedad, que impone restricciones en el abanico de expresiones posibles; por otro lado, los sujetos en tanto actores sociales, a través de la práctica (Ortiz y Delgado 2002) operan sobre estas expresiones, generando resignificaciones, reelaboraciones e incluso nuevas expresiones.

Teniendo en cuenta estos aportes, consideramos aquí al estilo cerámico en sentido amplio, como constituido por una serie de dimensiones -variables morfológicas, tecnológicas y decorativas integradas-, que definen un modo de hacer (Miller 1985; Rouse 1960), contextualizado social e históricamente, y producto de la conjunción de decisiones individuales y condicionantes sociales. Se trata de aquello que posibilita reconocer a un objeto individual como parte de un conjunto, asociado a determinados espacio, tiempo y personas (Miller 1985). Al respecto, Gell (1998) señala que los objetos con atributos estilísticos compartidos poseen como elemento común, además de determinada propiedad formal, un factor mayor relativo a su carácter de expresión de la cultura; es decir, los atributos estilísticos se asocian mediante un esquema de transferencia a los valores compartidos de una comunidad.

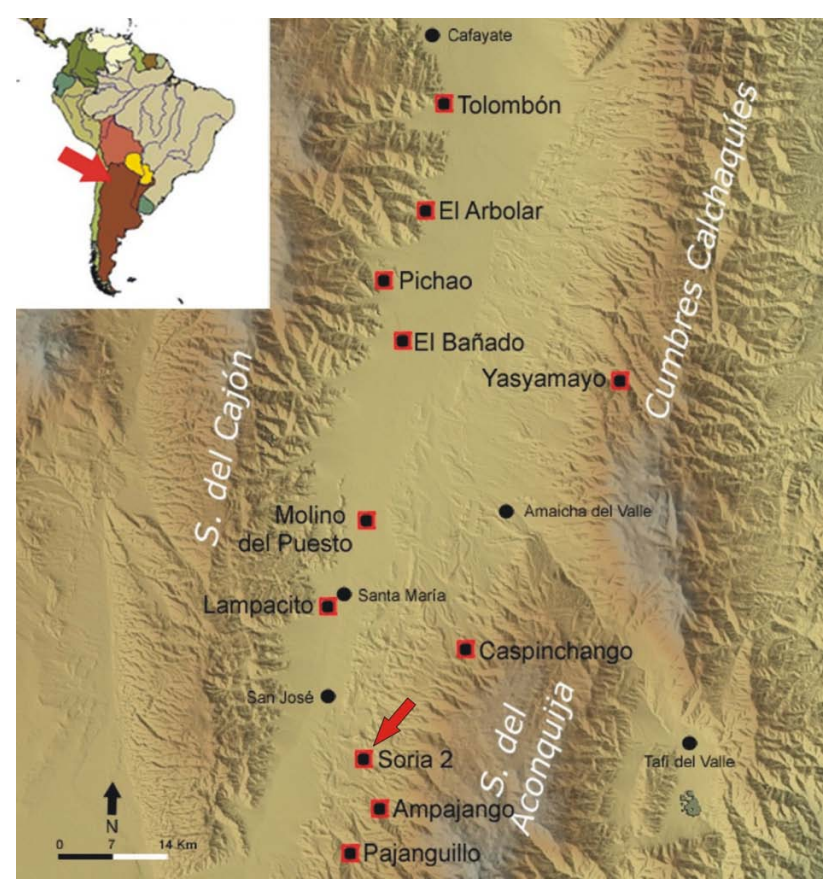

Figura 1. Mapa del valle de Yocavil, con ubicación del sitio Soria 2 y otros sitios formativos.

Figure 1. Yocavil valley map showing Soria 2 and other Formative sites location.

En el presente análisis, el empleo de la categoría estilo en sentido amplio se plasmó en la integración de las variables morfológicas, tecnológicas y decorativas relevadas, para clasificar el material en modalidades estilísticas y además como una primera instancia en el abordaje del estilo de vida de las poblaciones agropastoriles tempranas del área estudiada. Es necesario mencionar que la dimensión tecnológica referida a la petrografía de pastas no fue incluida en la propuesta de modalidades estilísticas en esta instancia, por las razones que se exponen en la sección correspondiente.

\section{El sitio Soria 2 y su cerámica}

En el sector oriental del sur del valle de Yocavil, entre el pie de la cadena principal del Aconquija y el río Santa María, se encuentra una antigua terraza del tercer nivel pedemontano de la formación Caspinchango, sobre cuyo sector meridional se ubica el poblado actual de Andalhuala-Banda. En la superficie de esta formación se emplazan estructuras arquitectónicas arqueológicas conformadas por uno o más recintos, líneas irregulares de piedras que definen amplias superficies aterrazadas, montículos y grandes rocas con morteros (Palamarczuk et al. 2007). Las características del terreno, sumadas a la cercanía a cursos de aguas permanentes, sugieren que se trató de una zona empleada para la producción agropecuaria en tiempos prehispánicos (Álvarez Larrain et al. 2009). La evidencia arquitectónica, así como los estilos de la cerámica de superficie, señalan una prolongada ocupación de este espacio, que se extiende 


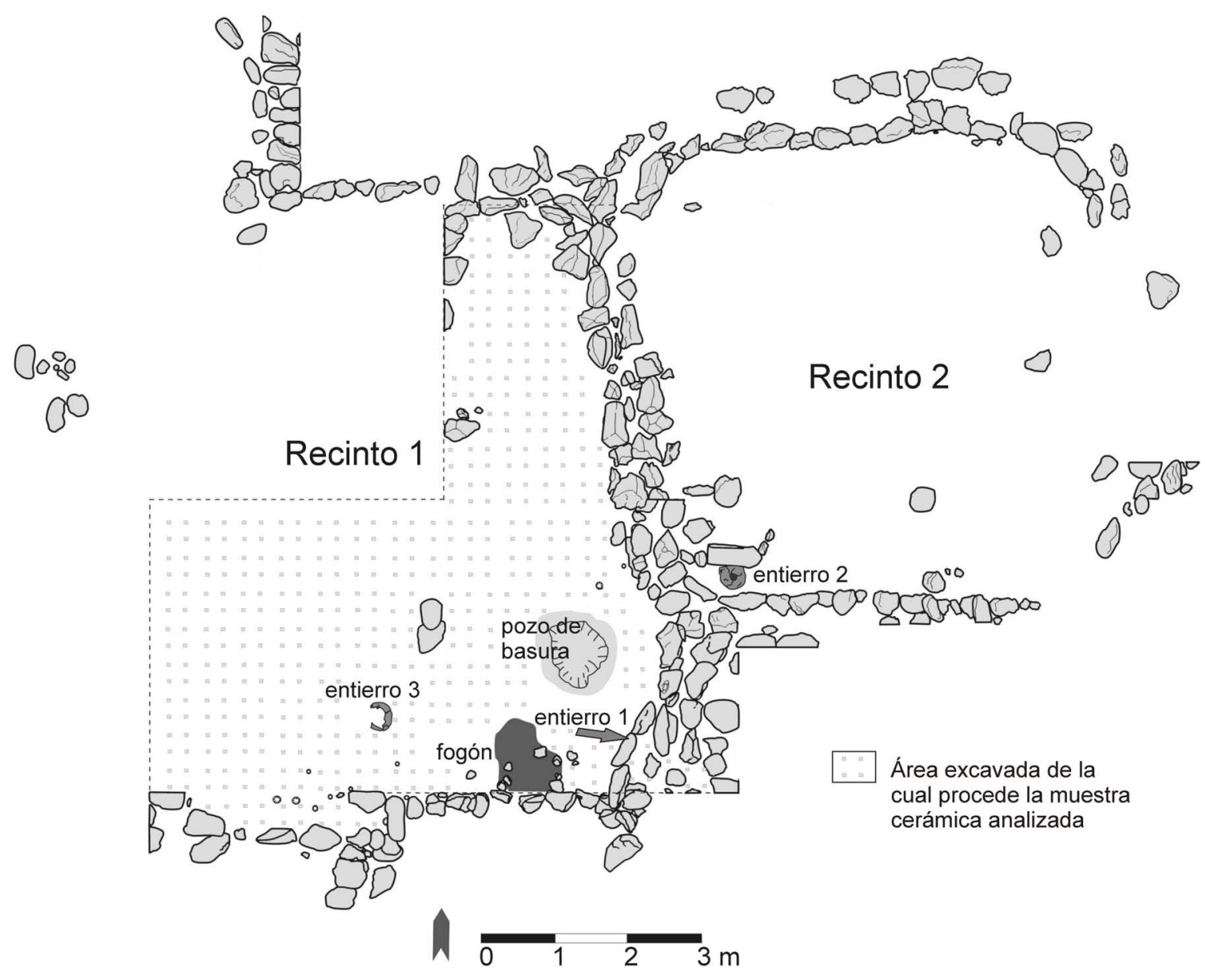

Figura 2. Planta del sitio Soria 2, con indicación del área excavada de la cual procede la muestra cerámica analizada.

Figure 2. Map of Soria 2 site. The doted area shows the excavated area.

hasta la actualidad. En base a la arquitectura remanente en superficie, se infiere que en algún momento de ese lapso el lugar presentaba una fisonomía aldeana; por el momento no se conoce fehacientemente el tipo de patrón de asentamiento involucrado. De este paisaje formaba parte el sitio Soria 2 (Figura 1).

La excavación del sitio, llevada a cabo en sucesivas campañas desde el año $2002^{1}$, permitió identificar hasta el momento al menos dos recintos adosados, denominados 1 y 2 (Figura 2). Se interpreta que se trata de un espacio destinado al uso doméstico, en función de los rasgos presentes en el piso de ocupación del recinto 1 -fogón, pozo destinado al descarte, agujeros de poste, etc.-, el cual es interpretado como un patio (Álvarez Larrain et al. 2009; Palamarczuk et al. 2007), y a los abundantes restos de piezas de alfarería encontrados, destinada a usos culinarios y de servicio (Baigorria Di Scala 2009; Spano 2008). En ciertos momentos del lapso de ocupación, este

\footnotetext{
${ }^{1}$ Campañas efectuadas en el marco del Proyecto Arqueológico Yocavil (Museo Etnográfico J. B. Ambrosetti, FFyL, UBA), dirigido por la Dra. M. Tarragó, y financiadas por los subsidios UBACyT F-018, ANPCyT PICT 04-12163, PID-CONICET 2218, PIP 6148, ANPCyT PICT 19-34511 y UBACYT F029.
}

patio pudo tener alguna clase de techado en el sector sur, considerando que se hallaron agujeros de poste a diferentes profundidades (Álvarez Larrain et al. 2009). Una muestra de carbones de la zona del fogón y sus inmediaciones arrojó un fechado de $1940 \pm 80$ ARCP (LP1541), es decir, 103 cal A.C. - 310 cal D.C. calibrados con dos sigmas. Esta cronología ubica al sitio en el Formativo o Temprano del NOA (extendido aproximadamente, entre el 600 A.C. y el 600 D.C. sensu Tarragó 1999).

Además de los indicadores de uso doméstico, en la vivienda se encontraron tres estructuras funerarias empleadas para el entierro de infantes; a cada una se le asignó un número, en base al orden en que las mismas fueron descubiertas. En el recinto 1 ocurrieron los denominados entierros 1 y 3 . El 1 corresponde a un entierro secundario: una línea simple de bloques de piedra cerraba la esquina SE del recinto, conformando un compartimento cerrado. Debajo de uno de esos bloques fue hallado parte del cráneo de un subadulto. A pocos centímetros, al interior del compartimento y directamente sobre tierra, se disponían restos esqueletales de al menos un subadulto, cubiertos con una mitad de olla de tipo ordinario (Spano et al. 2011). El entierro 3 es primario, 
se ubica en el sector sur del recinto y corresponde a la inhumación de un neonato contenido en una olla ordinaria (Palamarczuk et al. 2007).

El entierro 2, emplazado en el recinto 2, también de tipo primario, combina en cierta manera las modalidades de las otras dos inhumaciones: al interior de un compartimento abierto generado por un bloque de piedras situado en la esquina SO del recinto, se halló un neonato dentro de una olla ordinaria.

Aunque pertenecen al mismo período arqueológico (las piezas cerámicas asociadas comparten las características estilísticas de los conjuntos domésticos), los entierros son estratigráficamente posteriores a la formación del depósito de piso (Álvarez Larrain et al. 2009); los pozos correspondientes a las inhumaciones fueron excavados luego del uso de este espacio como área de vivienda.

En ambos recintos la densidad artefactual del piso fue notable; los restos culturales recuperados incluyen objetos de piedra (instrumentos tallados, desechos de talla, morteros, cuentas), artefactos sobre asta, huesos de fauna -mayormente pertenecientes a camélidos (Belotti L. de Medina 2007)-, entre otros elementos. Pero sobre todo, tanto el estrato de piso como el relleno del conjunto arquitectónico muestran un abrumador número de fragmentos cerámicos: hasta la campaña efectuada en 2010 se halló una cantidad que supera los 21.000; muchos evidenciaron ruptura in situ. El tamaño de los fragmentos fue variable, registrándose ejemplares que van desde $1 \mathrm{~cm}$ hasta $40 \mathrm{~cm}$ de largo máximo; el porcentaje de cada vasija representada por los fragmentos es variable, siendo el más común el $5 \%$, pero superando en algunos casos el $70 \%$.

Siguiendo la propuesta de un trabajo anterior (Baigorria Di Scala y Spano 2007), la alfarería del sitio es discriminada en dos grandes conjuntos: ordinario y fino. La alfarería ordinaria corresponde a piezas de pasta gruesa y porosa, con mayoría de inclusiones de tamaño mediano a grande, cocidas en atmósfera oxidante. Por conjunto fino aludimos a fragmentos de pasta compacta de baja porosidad, con inclusiones de tamaño pequeño o no distinguibles macroscópicamente; corresponde en su mayoría a piezas cocidas en atmósfera pobre en oxígeno.

Criterios semejantes fueron utilizados previamente en el estudio de cerámica de otros sitios tempranos del valle de Yocavil y áreas aledañas (Bugliani 2008, 2010; Bugliani y Pereyra Domingorena 1999). El estudio específico del conjunto ordinario ha sido encarado por Baigorria Di Scala (2009); aquí nos enfocaremos en el grupo de alfarería fina. En esta oportunidad presentamos el análisis efectuado sobre una muestra del conjunto fino correspondiente a ítems procedentes del contexto primario del recinto 1 , para el cual se cuenta con una superficie excavada de alrededor de $46 \mathrm{~m}^{2}$.

\section{Análisis de la alfarería fina del recinto 1}

En el estrato de piso del recinto 1 se contabilizaron 8707 fragmentos cerámicos; 25\% (N 2142) corresponde al conjunto fino.

Respecto al conjunto ordinario, mencionaremos algunas generalidades de referencia, remitiendo para mayor detalle a Baigorria Di Scala (2009). Se determinó la presencia de formas restringidas, básicamente ollas grandes de forma globular, halladas tanto en el piso de ocupación como asociadas a los entierros; se incluyeron además escudillas, pucos y cucharas. A la gran mayoría de estas piezas se les aplicó alisado en la superficie externa; también se hallaron algunos fragmentos pulidos con baño y engobe, peinados y pulidos regulares e irregulares. Las pastas son de compactación media a baja, con inclusiones de biotita, cuarzo y clastos líticos de origen granítico; la atmósfera de cocción es oxidante (Baigorria Di Scala 2009).

La muestra del conjunto fino de 2142 fragmentos, denominada muestra 1, fue inspeccionada para discriminar partes de vasijas (bordes, cuerpos, bases, asas; v. Tabla 1).

Teniendo en cuenta que la muestra presentaba un alto índice de fragmentación, el proceso de remontaje demandó un trabajo intenso y un tiempo prolongado de observación del material, poniendo en juego el cruce entre fragmentos procedentes de diferentes unidades estratigráficas (sensu Harris 1991).

\section{De la muestra 1 se acotó el universo muestreado a:}

- fragmentos diagnósticos en términos de forma (bordes, bases, asas, puntos característicos - de inflexión o angulares -)

- fragmentos con presencia de decoración

- fragmentos que remontan

Este procedimiento permitió conformar una muestra diagnóstica y representativa en términos de composición del universo total, denominada muestra 2, la cual fue abordada mediante el método de familias de fragmentos -FF- (Orton et al. 1997)2; a cada FF se le asignó un número identificatorio. Asimismo, se incluyeron en la cuantificación por FF, fragmentos de la muestra 1 que por sus características de pasta, tratamiento de la superficie y color presentaran similitudes lo suficientemente estrechas con materiales de la muestra 2, como para ser agrupados en FF. Esto dio un total de 569 fragmentos. La muestra 1 será abordada oportunamente.

\footnotetext{
2 Mediante este método se agrupan todos los fragmentos pertenecientes a una misma vasija (Orton et al. 1997); las agrupaciones en distintas familias responde a características macroscópicas de las pastas, formas, espesor de las paredes, color y tratamiento de la superficie, determinando así qué fragmentos del universo, potencialmente, podrían pertenecer a una misma vasija (Palamarczuk 2002).
} 


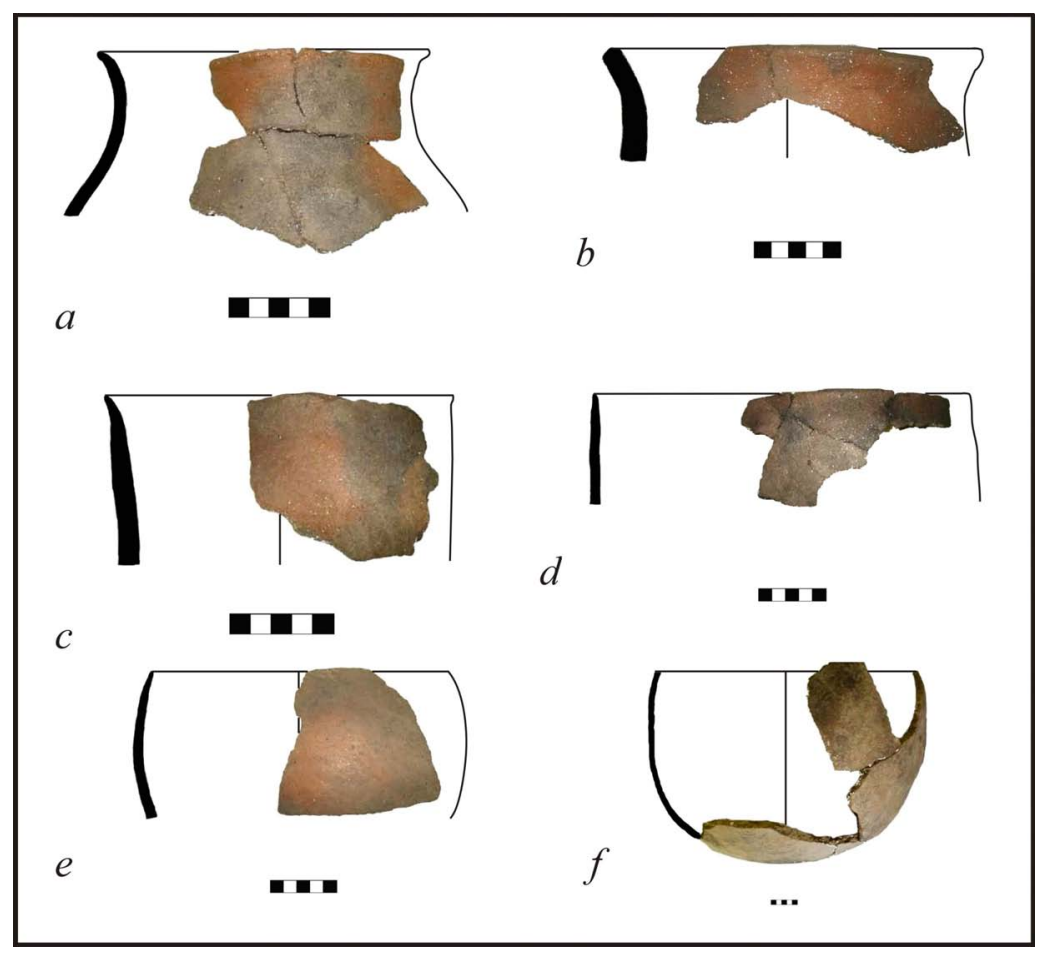

Figura 3. Vasijas del conjunto ordinario. $a$ y $b$ : ollas de contorno inflexionado; c: olla de contorno compuesto; $d$ : cuenco grande de contorno simple; $e$ : cuenco grande globular de perfil simple; $f$ : olla globular sin borde (elaborado a partir de Baigorria Di Scala 2009: 81 figura 54d; 81: figura 53b; 83: figura 56d; 74: figura 45a; 75: figura 46a; 84: figura 57 , respectivamente).

Figure 3. Ordinary group pottery. $a$ and b: pots with inflexed contour; c: pot with composite contour; $d$ : large bowl with simple contour; e: simple section globular large bowl; f: edgeless globular pot (modified from Baigorria Di Scala 2009: 81 figure 54d; 81: figure 53b; 83 : figure $56 \mathrm{~d}$; 74 : figure $45 \mathrm{a}$; 75 : figure 46a; 84: figure 57 .

por un solo fragmento (Figura 4); la familia con mayor cantidad de fragmentos alcanza los 38 (FF 205). Por otro lado, los datos deben leerse con miras a las intenciones que se persiguen al cuantificar un universo cerámico, esto es, determinar la composición de un conjunto dado (Orton et al. 1997).

\begin{tabular}{lc}
\hline Partes & $N^{\circ}$ de fragmentos \\
\hline bordes & 225 \\
asas & 8 \\
bases & 14 \\
cuerpos & 1894 \\
TOTAL & 2142 \\
\hline
\end{tabular}

Tabla 1. Partes de vasijas presentes en la muestra 1.

Table 1. Sample 1 portions of pottery.

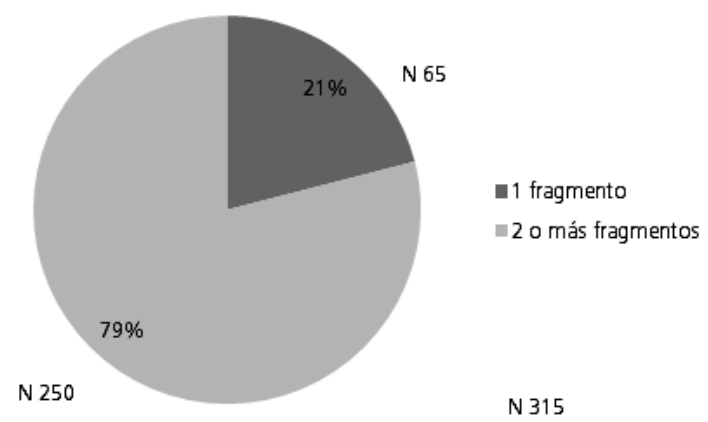

Figura 4. Distribución de FF según la cantidad de fragmentos que las componen.

Figure 4. FF distribution according to fragments frequency.

Como resultado del método de cuantificación aplicado a la muestra 2, se identificaron $315 \mathrm{FF}$. Si bien este número puede parecer en primera instancia alto, debe tenerse en cuenta el tamaño de la muestra de origen, y que un alto porcentaje está constituido por FF conformadas
El conjunto incluyó $11 \mathrm{FF}$ de estilos tardíos o de Desarrollos Regionales -900 al 1480 años D.C. (Tarragó 1999)-: Shiquimil, Santa María y Negro sobre Rojo Indeterminado. En todos los casos se trató de tiestos pequeños y de aristas muy desgastadas, lo cual nos lleva a interpretar que su procedencia original fue el estrato de relleno; la presencia detectada de perturbaciones causadas por roedores es un factor que pudo haber ocasionado la migración vertical de estos artefactos. El recinto 1, asimismo, no fue reocupado como unidad habitacional en tiempos tardíos, ya que no existe evidencia de pisos de ocupación para esos momentos ${ }^{3}$. Estos fragmentos son parte de la evidencia de un continuado uso del área en general por parte de las sociedades agroalfareras. Estas FF tardías no se consideraron en el análisis general, ya que el interés está puesto en caracterizar el universo correspondiente a las FF nativas del piso de ocupación (Harris 1991).

A continuación detallaremos los pasos seguidos para la construcción de las categorías de modalidades estilísticas.

\section{Formas}

La clasificación morfológica fue aplicada a aquellas FF correspondientes a contenedores cerámicos, es decir, a la vajilla utilizada en el recinto (no se incluyó en la contabilización a objetos no contenedores, como por ejemplo pipas).

El término puco se utiliza aquí como sinónimo de cuenco (sensu Balfet et al. 1992); esta categoría, en sus distintas

\footnotetext{
${ }^{3}$ En cambio, los mampuestos originales de los recintos fueron reutilizados en tiempos tardíos en la conformación de aterrazados de carácter probablemente agrícola (Palamarczuk et al. 2007).
} 


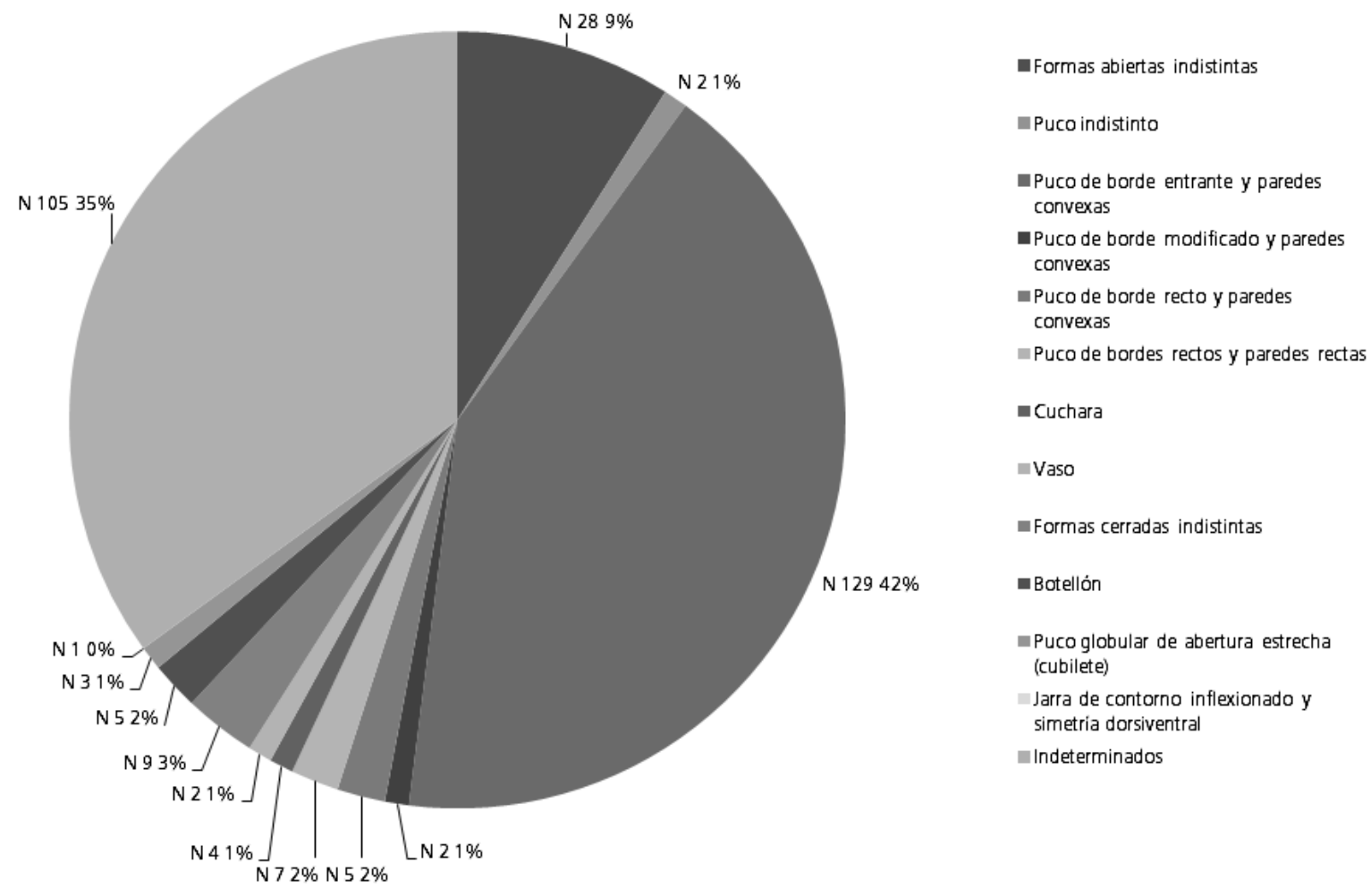

Figura 5. Porcentajes de formas presentes.

Resulta de interés revisar las categorías de bordes (Tabla 2), las cuales exhiben un predominio de bordes entrantes, en consonancia con la alta presencia de pucos. Es curiosa la detección de fragmentos de bordes ondeados (FF 47 y variantes, predomina por sobre el resto de las formas. Se agregaron categorías a aquellas planteadas en los esquemas clasificatorios generales (pucos globulares o cubiletes, jarras de contorno inflexionado y simetría dorsiventral), tomando en cuenta las clasificaciones morfológicas empleadas para el área del sur de los Valles Calchaquíes (Bugliani 2008), y denominaciones usadas para otras áreas del NOA (Tarragó 1989). Los porcentajes de formas identificadas se expresan en la Figura 5.

Dentro de la totalidad de formas, es llamativa la presencia de dos FF (\# 11 y 19) correspondientes a vasos cilíndricos de base plana y un asa vertical, similares a aquellos definidos por Krapovickas como de estilo Tebenquiche, de la Puna meridional catamarqueña (Krapovickas 1955). La categoría jarra de contorno inflexionado y simetría dorsiventral correspondió a la FF 184; si bien solamente se encontró parte de borde y cuello, su simetría es inferida por la curvatura del borde, la cual impide que la porción superior de la vasija genere sólidos de revolución (Shepard 1957); esta simetría posee laterales reflejos y frente y dorso distinguibles (Wolf y Kuhn 1977 [1952] citado en Scattolin 2006). No se identificaron escudillas; dado que suelen tener paredes delgadas, es probable que las mismas, de haber estado, hayan sufrido un alto índice de fragmentación (siguiendo esto, parte del 10\% de formas abiertas indistintas podrían corresponder a esta categoría). 174, v. Figura 6); si bien en un primer momento pensamos que podían pertenecer a otra clase de forma abierta (cucharas, por ejemplo), interpretamos que pertenecen a pucos de borde ondeado, de boca asimétrica.

Por fuera de la clasificación de contenedores cerámicos, encontramos dos FF correspondientes a pipas. La FF 27 es un fragmento de tubo; la FF 248 está conformada por medio hornillo tronco-cónico y el remache que adhiere parte de la base del mismo con el tubo; pertenece a una pipa de tipo acodado; fue hallada como basura primaria en el pozo de desechos del patio. Análisis químicos efectuados sobre los contenidos del hornillo identificaron la presencia de alcaloides compatibles con Anadenanthera colubrina var. cebil, indicando que se consumió dicha planta mediante prácticas fumatorias (Rosso y Spano 2005-2006).

\section{Técnicas de manufactura}

A partir de la revisión de las fracturas y de las superficies interna y externa de las piezas, se aprecia que la técnica predominante de modelado es la de rollos. Las fracturas son facetadas y las uniones de los rollos son visibles a ojo desnudo; la fragmentación de los tiestos se ha dado siguiendo las superficies de unión entre los rollos. Mediante esta técnica primaria (Rye 1981) se modelaron las FF que corresponden a las categorías puco (en todas 


\begin{tabular}{lc}
\hline Clases de bordes & Cantidad de FF \\
\hline entrantes & 174 \\
evertidos directos & 19 \\
rectos & 30 \\
ondeados & 2 \\
evertidos modificados & 1 \\
indeterminados & 9 \\
TOTAL & 235 \\
\hline
\end{tabular}

Tabla 2. Clases de bordes.

Table 2. Edge classes.

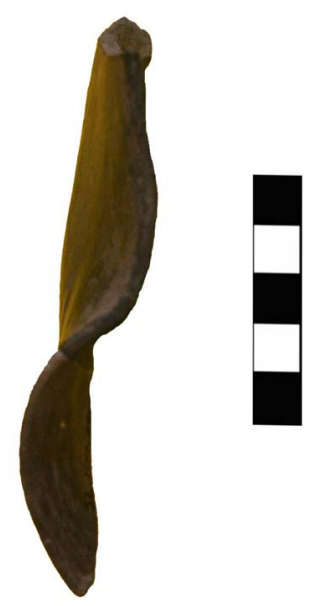

Figura 6. Fragmento de borde ondeado (FF 174)

Figure 6. Ondulated edge fragment (FF174).

sus variantes), vaso, botellón y jarra; adicionalmente, se recurrió a la técnica de pinching de manera secundaria, en la terminación de los bordes y en la aplicación de apéndices al pastillaje. Como técnica primaria se aplicó pinching en las FF que corresponden a las categorías cucharas o cucharones y pipas.

\section{Pastas}

Las pastas fueron analizadas a ojo desnudo y con lupa de 20 aumentos sobre fractura fresca. Las variables consideradas (siguiendo a Orton et al. 1997 y Palamarczuk 2002) fueron color de la pasta, inclusiones (tamaño, porcentaje, naturaleza y grado de desgaste), tipo de fractura y porosidad. Para la determinación de color se empleó la carta de colores Cailleux $(s / f)^{4}$.

Con la excepción de cinco FF, el resto posee pastas compactas finas, cuyas tonalidades van de gris claro a

\footnotetext{
4 La carta de colores Cailleux posee una alta correspondencia con la carta Munsell: adopta el sistema de codificación Munsell (basado en la combinación de tonos, valores y cromaticidades), pero consigna un rango de colores algo más acotado.
}

oscuro, e incluyen también tonos rojizos ${ }^{5}$. Las inclusiones en general aparecen en una proporción del 5 al 35\%, con tamaños de menos de 0,25 mm y de 0,25-0,50 mm; la naturaleza de las mismas corresponde mayormente a cuarzo, biotita y muscovita, y se presentan de forma subangular o indeterminada (con lo cual el grado de desgaste parece ser bajo). La fractura es subconcoidal. La porosidad aparente fue baja en general (alrededor del $6 \%)$.

Por otra parte, cuatro FF (\#183, \#184, \#205, y \#291) presentan pasta mediana, de tonalidades anaranjadas ${ }^{6}$; en todos los casos se trata de fragmentos de piezas de cocción oxidante. Poseen una proporción de inclusiones mayor al 10\%, de tamaño mediano (de 0,25 mm a $0,50 \mathrm{~mm}$ ); incluyen cuarzo, biotita y minerales ferrosos, de forma subangular o redondeada (con un grado de desgaste de medio a alto). La fractura es irregular. La porosidad aparente fue de alrededor del $10 \%$.

A los fines de lograr una primera aproximación más ajustada a la caracterización de las pastas, se realizó el análisis petrográfico de cortes delgados con microscopio de polarización, de una pequeña muestra constituida por cinco fragmentos, que pudiera servir de base para la planificación de un número mayor de cortes (Spano 2008). La información petrográfica no fue incluida en la propuesta de modalidades estilísticas, debido a la reducida muestra con la que contamos por el momento.

\section{Cocción}

El $88 \%$ de las FF corresponde a fragmentos de piezas cocidas en atmósfera pobre en oxígeno. En general la cocción se dio de manera despareja; son visibles variaciones en la tonalidad superficial aun en una misma pieza, debido al ingreso de aire en la atmósfera de cocción, ocasionando manchas parduscas o rojizas; esta falta de homogeneidad puede ser atribuible a las condiciones de control del horno durante el proceso de cocción de las piezas. En este sentido, los análisis de cortes delgados de piezas grises mostraron señales de elementos oxidantes, como color pardo del núcleo, lo cual estaría indicando que la atmósfera de cocción no fue completamente reductora (Spano 2008). Esta cocción dio como resultado pucos, vasos, botellones y cucharas de color negro, negro grisáceo y gris ${ }^{7}$.

El $12 \%$ restante fue logrado mediante cocción en atmósfera oxidante y oxidante incompleta, obteniéndose piezas de tonalidades ante, anaranjada y rojiza ${ }^{8}$,

${ }^{5}$ Gris claro: 7,5R 7/0 Gris y 7,5 R 6/0 Gris. Gris oscuro: 2,5 Y 5/0 Gris; 7,5 R 4/0 Gris oscuro; 5 Y 3/1 Gris muy oscuro. Rojizos: 5 YR 6/6 Amarillo rojizo; 7,5 YR 7/4 Rojo.

610 R 6/8 Rojo claro; 5 YR 5/4 Marrón rojizo.

${ }^{7} 2,5$ Y 3/0 Gris muy oscuro; 7,6 R 4/0 Gris oscuro; 2,5 Y 4/0 Gris oscuro; 7,5 R 6/0 Gris; 2,5 Y 5/0 Gris; 10 YR 6/1 Gris y 2,5 Y 4/0 Gris oscuro.

85 YR 6/3 Marrón rojizo claro; 5 YR 6/4 Marrón rojizo claro; 5 YR 7/4 


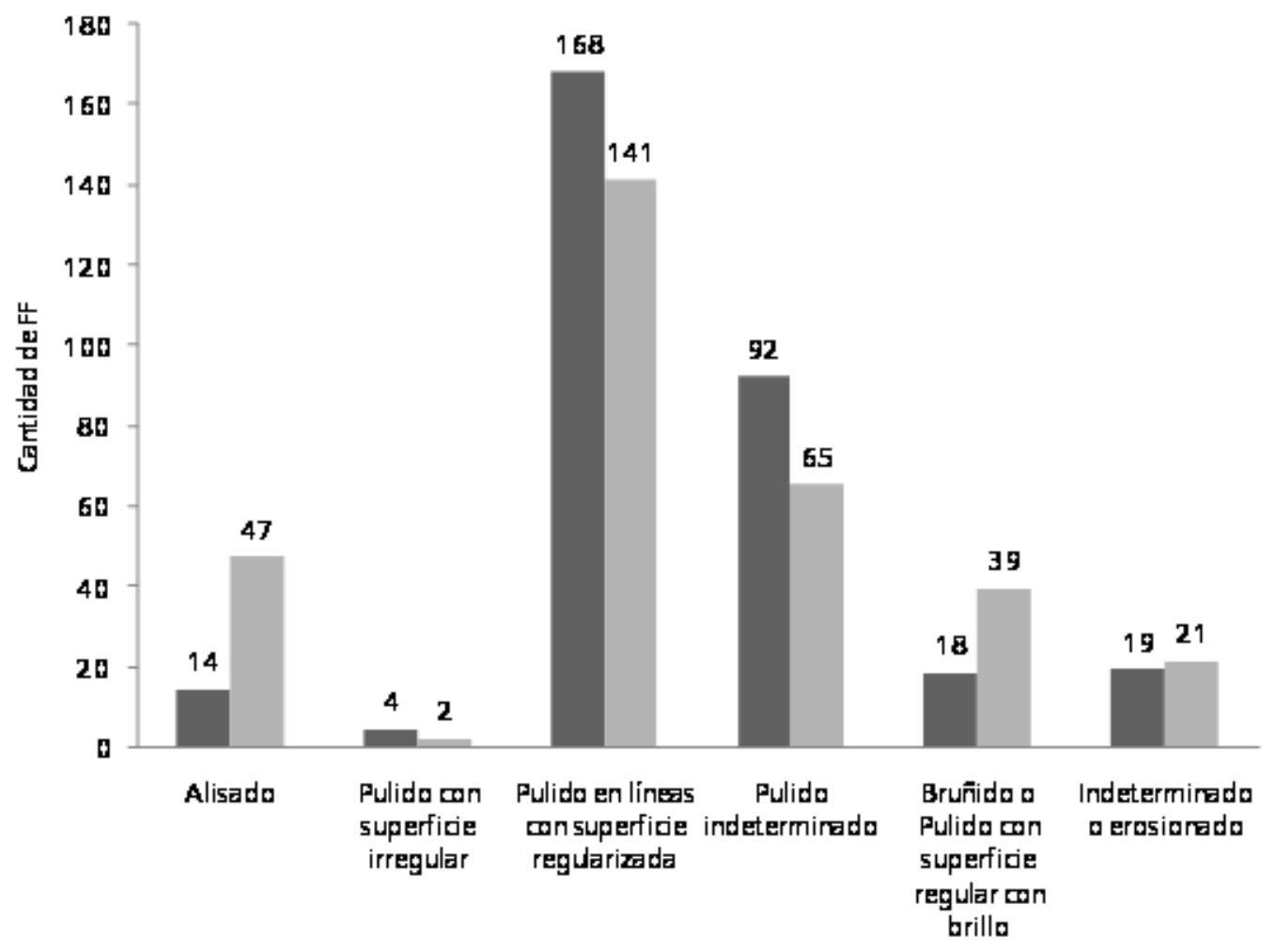

Tratamiento dela superficie

Tatal315

Figura 7. Tratamiento de la superficie, distribuido por FF.

Figure 7. Surface treatment, sorted by FF.

que corresponden a pucos, botellones y jarras. Estas tonalidades englobadas bajo los colores habitualmente denominados ante o marrón rojizo, son claramente diferenciables de los colores ladrillo de la cerámica obtenida en atmósfera oxidante en momentos tardíos en el Valle, en cuyo proceso de manufactura se contaba con un control mayor sobre las condiciones de cocción.

\section{Tratamiento de la superficie}

El acabado o tratamiento de la superficie tiene el objeto de regularizar imperfecciones y marcas dejadas por el artesano durante la etapa de modelado, y de delinear el contorno de la pieza (Shepard 1957). Asimismo, según las técnicas que se utilicen, se le puede otorgar a la pieza propiedades favorables a nivel funcional, como impermeabilidad en las superficies internas mediante el pulido; y al mismo tiempo, texturas particulares a nivel estético, que inciden muchas veces en las definiciones de modalidades estilísticas e intervienen en los aspectos expresivos de la alfarería.

El tratamiento predominante es el pulido. En menor medida, se utilizó el alisado.

-Se definieron tres clases de pulido:

Rosa; 5 YR 5/4 Marrón rojizo; 7,5 YR 4/2 Marrón oscuro y 5 YR 4/2 Gris rojizo oscuro.
- pulido con superficie irregular: se notan las líneas de pulimento y se perciben sectores que no han sido tratados, con irregularidades en la superficie

- pulido en líneas con superficie regularizada: se advierten las líneas de pulimento, sobre una superficie que ha sido regularizada; las líneas varían en su grosor (desde $3 \mathrm{~mm}$ hasta $6 \mathrm{~mm}$ )

- pulido con superficie regular con brillo o bruñido: no son visibles las líneas de pulimento; las superficies se muestran con un regularizado parejo y aspecto lustroso - pulido indeterminado: se advierte presencia de pulido, pero por la erosión sufrida por los fragmentos no es posible asignar los mismos a ninguno de los otros tres grupos

Como se observa en la Figura 7, existe una cierta tendencia de paridad en la aplicación de pulido tanto en las superficies externas como internas de las vasijas. En el caso de la modalidad de pulido en líneas con superficie regularizada, se notó una tendencia hacia los trazados horizontales en las superficies externas de los pucos, dando como resultado líneas paralelas o subparalelas entre sí; en los vasos, botellones y jarras, en cambio, los trazados son verticales en la superficie externa, siguiendo el eje mayor de la pieza. En otros casos, el pulido se efectuó en sentido cruzado diagonal ("en $\mathrm{X}^{\prime \prime}$ ), dando como resultado líneas superpuestas y una textura reticulada en la superficie interna de pucos, que 

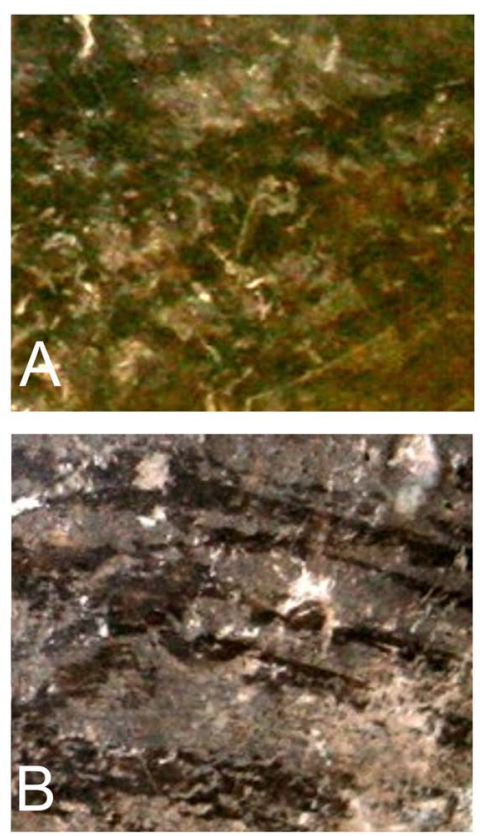

Figura 8. A: detalle de pulido en líneas conformando una textura reticulada; B: detalle de pulido en líneas en sentido horizontal.

Figure 8. A: detail of linear polish forming a reticulated texture. B: detail of horizontally linear polish

contrasta con el efecto del pulido en líneas horizontales de la superficie externa (Figura 8).

Solamente seis FF presentaron aplicación de baño, de color morado (un caso), ante (dos casos) y de color igual a la pasta (tres casos).

\section{Decoración}

Un 26\% de las FF evidenció la ejecución de técnicas decorativas, entendiendo por las mismas el modelado, la incisión, el grabado y la pintura. Estas técnicas también podrían ser consideradas como parte del tratamiento de la superficie, sin embargo fueron distinguidas por ser las mismas el vehículo de expresión de los elementos de diseño. Las técnicas detectadas y sus respectivas proporciones por FF se expresan en la Figura 9.

La técnica de pintura fue diferenciada según el momento en el cual la misma fue aplicada, respecto de la instancia de cocción. Se registró pintura previa a la cocción en la FF 304, de estilo Vaquerías (Figura 10a). Por pintura post cocción aludimos a la aplicación de un pigmento de color rojo intenso sobre la superficie de la vasija ya cocida, el cual, por no haber sido expuesto a temperaturas elevadas, no se fija a la pieza como en el caso de la pintura previa a la cocción (Haber 2006). Se presentó en dos casos; por un lado, la FF 248, correspondiente a una pipa en cuyo hornillo se ejecutaron líneas en zig-zag en rojo, en combinación con pintura negra, sumamente desvaída (Figura 10b). En segundo término, en la FF 249, que

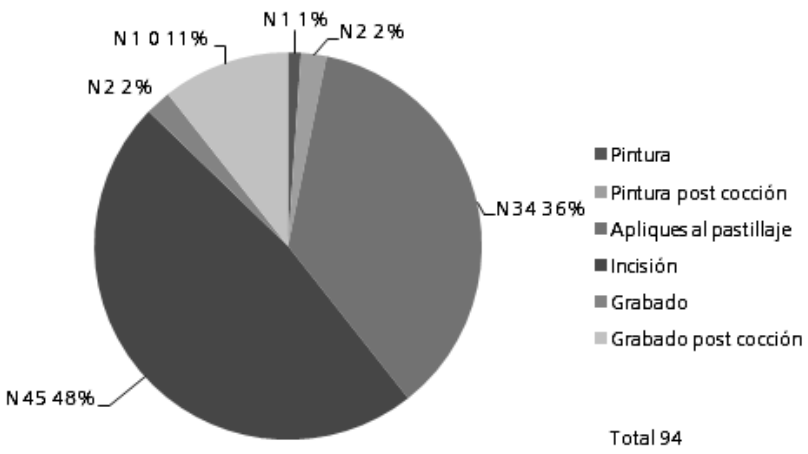

Figura 9. Técnicas decorativas presentes por FF.

Figure 9. Decorative techniques by FF.

corresponde a un fragmento de puco sobre el que se trazaron líneas superpuestas en el borde interno; esta pieza fue hallada en el espacio del entierro 1 (véase Figura 14c, más adelante).

El grabado precocción y la incisión fueron aplicados utilizando instrumentos de puntas simples. Se ejecutaron en formas cerradas, con la salvedad de la FF 260, la cual corresponde a un puco de paredes rectas; en otros casos, no se pudo determinar forma para FF que presentaban incisión (Figura 10c, 10d y 10e).

En el caso de las FF 184 y 205, la decoración por aplicación de pastillaje y la incisión se combinaron en la conformación de motivos complejos, los cuales representan rostros (antropomorfo y zoomorfo, respectivamente), ejecutados sobre el borde y el cuello en el primer caso, y sobre el cuerpo de la vasija en el segundo (Figura 10f y 10g). Se trata de dos vasijas efigie o prosopomorfas, es decir, recipientes en los cuales la forma está involucrada en el referente que se quiere representar.

Asimismo, se registró una llamativa modalidad de ejecución de grabado post cocción en la superficie interna de formas abiertas. El mismo se manifiesta claramente por las marcas de saltado dejadas por el instrumento utilizado, y la falta de control sobre los trazos, los cuales son desprolijos. Los fragmentos que presentaron esta modalidad decorativa en la muestra analizada corresponden a dos FF de cocción oxidante incompleta, y a ocho FF de cocción en atmósfera pobre en oxígeno; en todos los casos pertenecen a pucos, formas que brindan la posibilidad de ejecutar esta particular técnica por el espacio disponible para el artesano (Figura 10h). En este caso se puede apreciar la imbricación entre lo tecnológico y lo decorativo: la aplicación de una determinada técnica en un momento dado de la cadena operativa, a la vez, puede estar generando un recurso estilístico.

Los apliques al pastillaje modelados en pucos constituyen la técnica más popular de la muestra; se trata de aditamentos que conforman por sí solos unidades de 


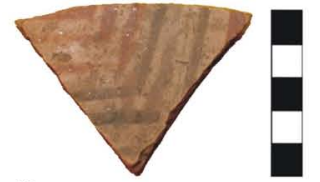

$a$

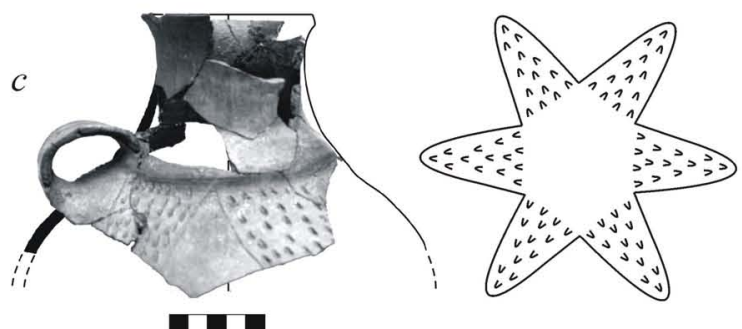

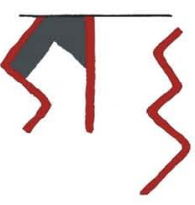

$b$

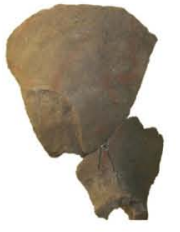

$d$

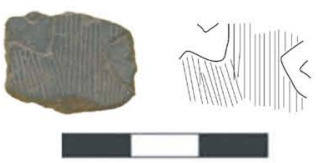

e

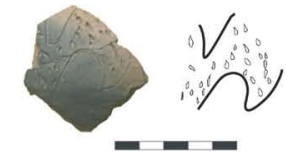

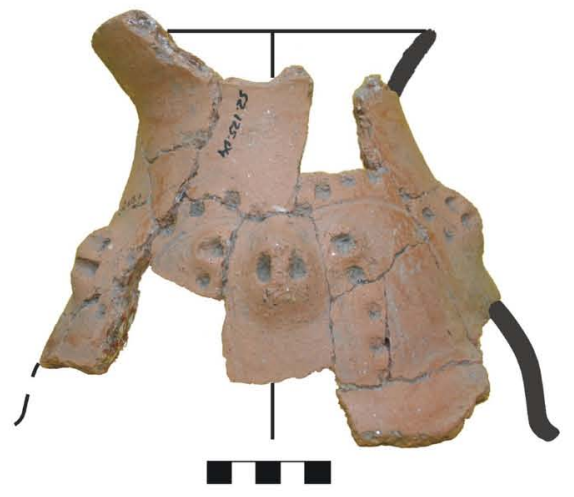

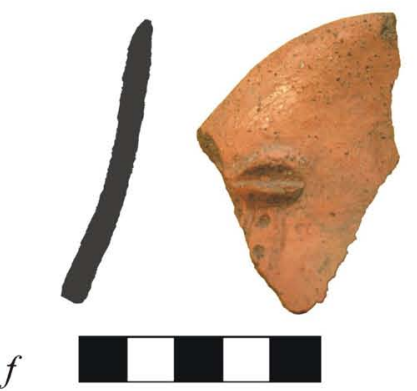

$g$
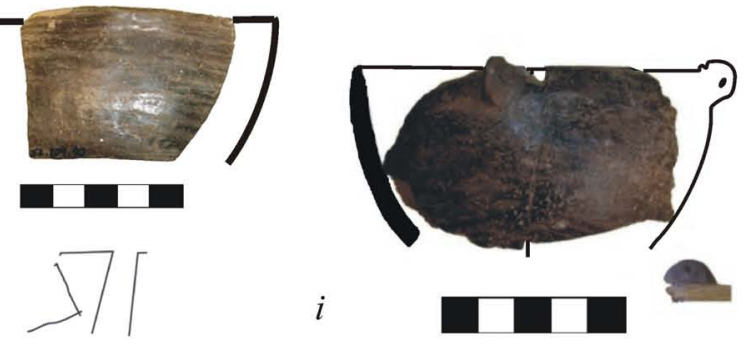

Figura 10. Distintas técnicas decorativas. $a$ : fragmento Vaquerías -FF 304-, aplicación de pintura. $b$ : pipa con despliegue de diseños -FF 248-, aplicación de pintura post cocción muy desvaída; c: botellón inciso, con reconstrucción y despliegue de diseños (vista superior) -FF 216-; d: fragmento grabado -FF 260-; $e$ : fragmento inciso -FF 285-; $f$ : jarra prosopomorfa de contorno inflexionado y simetría dorsiventral, con apliques al pastillaje e incisión que conforman un rostro antropomorfo -FF 184-; $g$ : botellón con apliques al pastillaje e incisión que conforman un rostro zoomorfo -FF 205-; $h$ : puco con grabado post cocción en la superficie interna -FF 165-; $i$ : puco con apliques al pastillaje sobre el borde, conformando una cabeza zoomorfa esquemática -FF 155-.

Figure 10. Different decorative techniques. a: Vaquerias fragment-FF304- paint application. b: pipe with design deployment -FF248-, application of post firing paint; c: incise bottle with reconstruction and design deployment (upper view) -FF216-; d: engraved fragment -FF260 -; e: incise fragment -FF 285-; $f:$ prosopomorphic jar with inflexed contour and dorsiventral symmetry, showing pastillage appliqués and incision forming an anthropomorphic face -FF 184-; g: bottle with pastillage appliqués and incision forming a zoomorphic face -FF205-; h: bowl with post firing engraving in inner surface-FF165-; i: bowl with pastillage appliqués on the edge forming and schematic zoomorphic head -FF155- diseño: los artesanos optaron por aplicar protuberancias cónicas ó subesferoidales, adheridas a los labios; las mismas pueden presentar incisiones, de modo de mostrar una variedad de representaciones de acuerdo a la clase de trazo (líneas cortas, puntos) y al número de los mismos en cada aditamento (Figura 10i). A su vez, estos apliques pueden ser dobles. De este modo se lograron elementos de diseño no figurativos y figurativos: zoomorfos naturalistas -en apariencia, cuerpos y cabezas de murciélagos, roedores o cánidos y batracios-, y zoomorfos no naturalistas o esquemáticos, es decir, rostros cuyos componentes se representan apelando a su mínima expresión; en algunos casos, se presentan sin ojos (Figura 11). Estos recursos plásticos han sido reportados para Bañado Viejo en el norte del valle de Yocavil (Bugliani y Pereyra Domingorena 1999), en Loma Alta -falda occidental del Aconquija- y en Yutopián, en el valle del Cajón (Bugliani 2008).

Se registró el repertorio de elementos decorativos o motivos de la muestra, apuntando a reconocer las representaciones aludidas por los objetos cerámicos. Una vez identificados los elementos decorativos, fueron clasificados en figurativos (representaciones de objetos identificables y/o biomorfos) y no figurativos (representaciones en las cuales se desconoce el referente objetivo) (Aschero 1988). Esta clasificación se expresa en la Tabla 3.

La determinación de los elementos de diseño estuvo acompañada por la definición de campos decorativos, es 


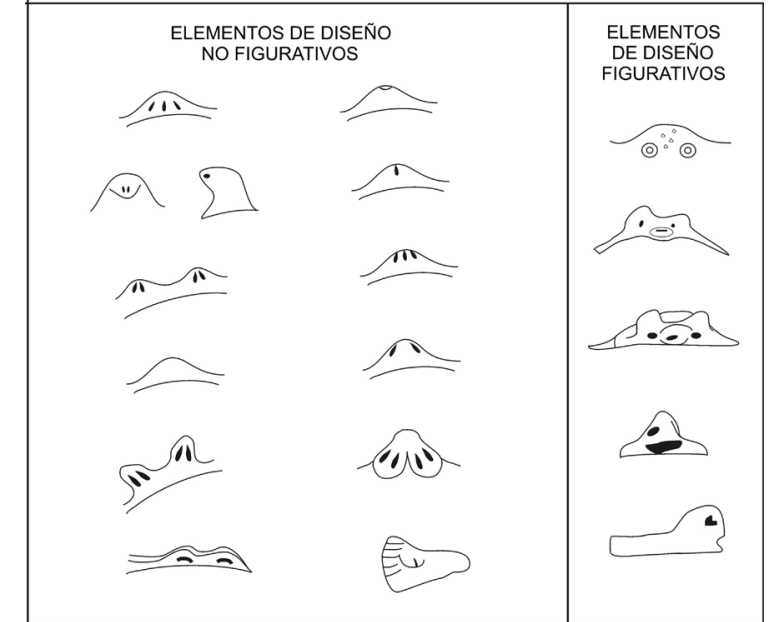

Figura 11. Variedad de apliques modelados al pastillaje presentes en pucos.

Figure 11. Variety of pastillage appliqués identified in bowls.

Tabla 3. Técnicas de ejecución y elementos de diseños.

Table 3. Techniques and design elements.

\begin{tabular}{|c|c|c|}
\hline Técnica & Elemento de diseño no figurativo & Elemento de diseño figurativo \\
\hline \multirow{6}{*}{ grabado } & líneas paralelas & \\
\hline & campos romboidales rellenos con líneas paralelas & \\
\hline & punto & \\
\hline & línea recta & \\
\hline & línea curva & \\
\hline & zig-zag & \\
\hline \multirow{10}{*}{ grabado post cocción } & chevrón & \\
\hline & líneas paralelas & \\
\hline & espigado & \\
\hline & cruz doble & \\
\hline & línea ondulada & \\
\hline & líneas paralelas & \\
\hline & zig-zag & \\
\hline & chevrón & \\
\hline & líneas oblicuas cruzadas entre sí & \\
\hline & puntos no alineados & \\
\hline \multirow{10}{*}{ incisión } & campos triangulares rellenos con puntos arrastrados & \\
\hline & puntos entre líneas curvas & \\
\hline & círculos entre líneas curvas & \\
\hline & puntos en apéndices cónicos & \\
\hline & puntos arrastrados en apéndices cónicos & \\
\hline & volutas rellenas con puntos arrastrados & \\
\hline & apéndice cónico/subesferoidal & zoomorfo naturalista \\
\hline & apéndice cónico/subesferoidal & \\
\hline & con puntos incisos & \\
\hline & apéndice cónico/subesferoidal con puntos arrastrados & zoomorfo / biomorfo esquemático \\
\hline \multirow{5}{*}{$\begin{array}{l}\text { aplicaciones al pastillaje y } \\
\text { modelado }\end{array}$} & apéndice chato con líneas paralelas & \\
\hline & botón elongado & \\
\hline & botón con puntos incisos & ojo en grano de café \\
\hline & ojo en grano de café & \\
\hline & puntos & \\
\hline pintura & chevrón & \\
\hline \multirow{2}{*}{ pintura post cocción } & zig-zag & \\
\hline & líneas irregulares & \\
\hline
\end{tabular}




\begin{tabular}{lll}
\hline Forma & Técnica de ejecución & \multicolumn{1}{c}{ Campo decorativo } \\
\hline $\begin{array}{l}\text { pucos y formas abiertas } \\
\text { indeterminadas }\end{array}$ & $\begin{array}{l}\text { aplicación al pastillaje } \\
\text { pintura post cocción } \\
\text { pucos restringidos }\end{array}$ & $\begin{array}{l}\text { borde externo y sector superior externo del cuerpo } \\
\text { sector superior externo del cuerpo (extensión indeterminada) }\end{array}$ \\
\cline { 2 - 3 } botellones & aplicación al pastillaje & borde externo y sector superior externo del cuerpo \\
\cline { 2 - 3 } jarras prosopomorfas & incisión & sector superior externo del cuerpo \\
pipas & aplicación al pastillaje & cuello y sector superior externo del cuerpo \\
\cline { 2 - 3 } & aplicación al pastillaje & cuello (extensión indeterminada) \\
\cline { 2 - 3 } & $\begin{array}{l}\text { pintura post cocción } \\
\text { incisión }\end{array}$ & superficie externa del hornillo \\
\end{tabular}

Tabla 4. Campos decorativos según forma y técnica de ejecución de los diseños.

Table 4. Decorative fields by shape and design techniques.

decir, el establecimiento del sector de la pieza que ha sido decorada (Shepard 1957); de este modo, se abordaron como dos aspectos integrados la forma y la decoración. Este objetivo se vio limitado en parte por la fragmentación de la muestra, no obstante lo cual pudieron definirse algunas recurrencias, expresadas en la Tabla 4.

\section{Modalidades estilísticas}

Teniendo en cuenta lo referido en la sección anterior, se realizó una clasificación por modalidades estilísticas de la muestra 2, considerando los atributos formales, tecnológicos y decorativos de las FF, a los fines de contar con un primer criterio que permita ordenar la variabilidad presente y además, constituya una guía para el análisis de la cerámica del sitio y del área de Andalhuala-Banda. El material analizado en este estudio es fragmentario y, en un punto, incompleto (por las restricciones de la muestra y por el hecho de que no se concluyó la excavación del recinto 1); por lo tanto, no sería adecuado realizar una clasificación en la cual la aplicación de técnicas, por ejemplo la incisión, incidiera en la definición de categorías diferenciadas, debido a que fragmentos lisos (sin incisión) pudieron formar parte de campos no decorados de vasijas incisas. Consecuentemente, se proponen categorías amplias, las cuales puedan ser afinadas en la medida en que avance el proceso de estudio de esta alfarería. Las particularidades introducidas por el tratamiento de la superficie y la decoración fueron consignadas como un elemento más de la caracterización, a fin de registrar su presencia o ausencia. Asimismo, se tuvieron en cuenta las designaciones para estilos conocidos, según la bibliografía consultada. Esta primera clasificación se basó en aquellas FF que permitieron un remontaje mayor, así como en FF de un solo integrante diagnóstico en términos de forma o atributos decorativos, a partir de comparaciones con vasijas enteras procedentes de colecciones, relevadas por nosotros o aludidas por otros autores (Bugliani 2008; Scattolin y Bugliani 2005); este criterio comprometió a 89 FF. En el gráfico plasmado en la Figura 12 se informa acerca de los porcentajes de FF en que se expresan las modalidades propuestas.

En relación a las características petrográficas de las pastas, esperamos que la realización de nuevos estudios de cortes delgados con la consiguiente ampliación de la muestra, nos permita integrar un análisis de pastas más detallado a la propuesta planteada.

Las modalidades estilísticas resultantes fueron:

A) Alfarerías plomizas pulidas: engloba al grupo obtenido en atmósfera de cocción pobre en oxígeno, que constituye la mayor parte de la muestra. Las pastas son finas y compactas. Presenta tonalidades 2,5 Y 5/0 Gris; 7,5 R 6/0 Gris; 10 YR 6/1 Gris; 2,5 Y 4/0 Gris oscuro; 7,6 R 4/0 Gris oscuro; 2,5 Y 3/0 Gris muy oscuro; 7,6 R 4/0 Gris oscuro; y 2,5 Y 4/0 Gris oscuro. Las formas incluyen:

- pucos no restringidos de contorno simple

- pucos restringidos de contorno simple

- botellones

- cucharas

Se registró el uso de incisión, grabado, grabado post cocción, pulido en líneas y pulido con superficie regular con brillo o bruñido (éste último solamente en pucos); pueden presentar modelados y apliques al pastillaje (Figura 13a).

B) Alfarerías café pulidas: alude a piezas de cocción oxidante incompleta, con coloraciones que van desde el ante hasta el marrón rojizo (5 YR 6/3 Marrón rojizo claro; 5 YR 6/4 Marrón rojizo claro; 7,5 YR 4/2 Marrón oscuro; y 5 YR 4/2 Gris rojizo oscuro). Las pastas son finas y compactas. Las formas incluyen:

- pucos no restringidos de contorno simple

- pucos restringidos de contorno simple

Se aplicó la incisión, el grabado post cocción, la pintura post cocción y el pulido en líneas; pueden presentar asimismo modelados y apliques al pastillaje (Figura 13b).

C) Alfarerías alisadas: dado que el desgaste producido por el paso del tiempo sobre los fragmentos puede ocasionar la pérdida del tratamiento de la superficie original, se tuvo cuidado al momento de considerar los casos alisados. 


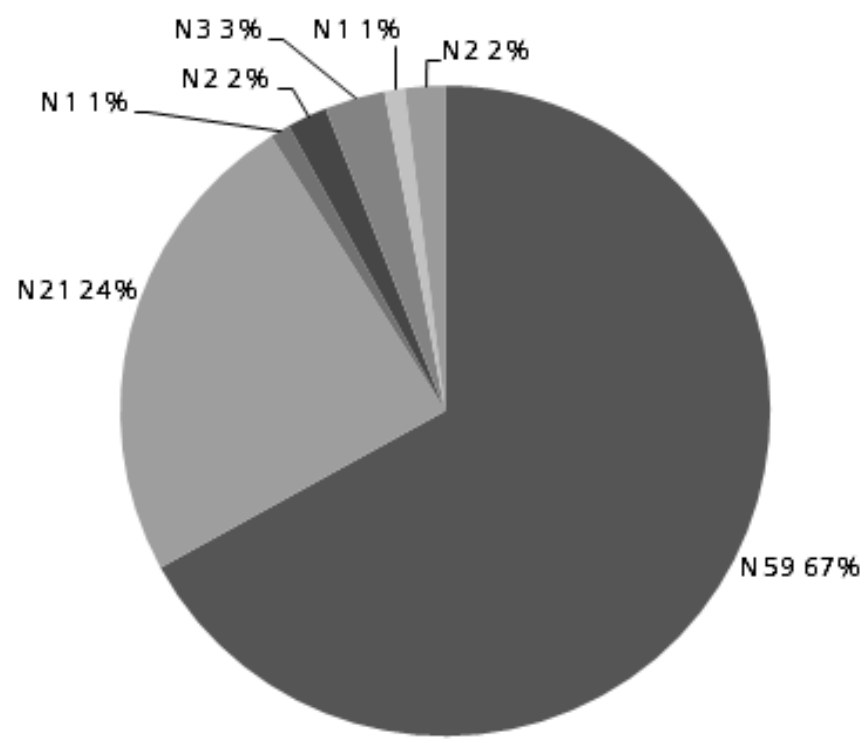

\author{
- Alfarerías plomizas pulidas \\ - Alfarerias cafépulidas \\ "Alfarerías alisadas \\ -Alfarerias naranja pulidas de \\ pastafina \\ Alfarerías maranja pulidas de \\ pasta mediana \\ Vaquerias \\ - Tebenquiche
}

Figura 12. Porcentajes de modalidades estilísticas definidas, en base a las familias de fragmentos consideradas.

Figure 12. Frequency of stylistic modes, based on considered fragment families.

Por lo tanto, esta categoría se basó en una sola FF, que brindaba la seguridad para esta afirmación. Se trata de una pieza obtenida en atmósfera de cocción pobre en oxígeno, de pasta fina y compacta; la tonalidad es 2,5 Y $5 / 0$ Gris. La forma corresponde a un puco no restringido de contorno simple (Figura 13c).

D) Alfarerías naranja pulidas de pasta fina: tanto esta categoría como la siguiente, se diferencian de las alfarerías pulidas café por presentar una atmósfera de cocción claramente oxidante, originando tonalidades anaranjadas en la cerámica. La pasta es fina y compacta, puede presentar pulido en líneas y aplicación de baño. Poseen tonalidad 5 YR 5/4 Marrón rojizo. No fue posible definir formas, aunque se alcanzó a reconocer formas cerradas indeterminadas (Figura 13d).

E) Alfarerías naranja pulidas de pasta mediana: a diferencia de la categoría anterior, en este caso las pastas son de textura media y poco compactas, con inclusiones de mayor tamaño. Presentan tonalidad 5 YR 7/4 Rosa. Las formas corresponden a:

- botellones

- jarras prosopomorfas o efigies de simetría dorsiventral

Se empleó la combinación de decoración por modelado al pastillaje y la incisión. Esta modalidad guarda semejanzas con el estilo definido como Candelaria I fase El Mollar o Tafí I (González 1960; Heredia 1974)9 (Figura 13e).

\footnotetext{
${ }^{9}$ A su vez, se ha señalado la existencia de ciertas afinidades estilísticas entre Candelaria I fase El Mollar / Tafí I, y alfarería hallada en sitios de la tradición San Francisco (Garay de Fumagalli y Cremonte 2002; González 1960; Heredia 1974).
}

F) Estilos definidos con anterioridad: Tebenquiche (Krapovickas 1955); Vaquerías (Heredia et al. 1974; Korstanje 1998) (Figuras $13 f$ y $10 a$, respectivamente).

\section{Objetos cerámicos y prácticas}

En Soria 2, la recuperación de material en posición primaria constituye una ventana al pasado con miras a relevar las prácticas en las cuales las vasijas estuvieron involucradas; en otra oportunidad se expondrá la puesta en juego de la clasificación de modalidades estilísticas en vinculación con información contextual y evidencia directa. A los fines de complementar la presente propuesta, resumimos algunos de esos planteos en lo que refiere al recinto 1.

Como mencionáramos líneas arriba, se interpreta al recinto 1 como un patio; como tal, implicó un ámbito de circulación cotidiana de un número determinado de personas que residían en la vivienda, y la práctica recurrente en el tiempo de actividades; de este modo, las rutinas diarias construían el proceso de socialización de los sujetos (Bourdieu 1977); las vasijas finas participaron de esta reproducción social.

Este conjunto se distribuyó homogéneamente en el patio, estando representadas todas las modalidades estilísticas; a la vez, fragmentos pertenecientes a una misma FF se hallaron concentrados en determinados puntos, señalando los lugares de ruptura in situ de las vasijas a las cuales pertenecieron. Se puede plantear, por un lado, que existió una categoría de FF correspondientes a objetos que intervinieron en situaciones cotidianas, que se manifiestan en los restos dispuestos en el piso de ocupación y el pozo de basura; corresponden a piezas que se han reconstruido en porcentajes diversos, desde 

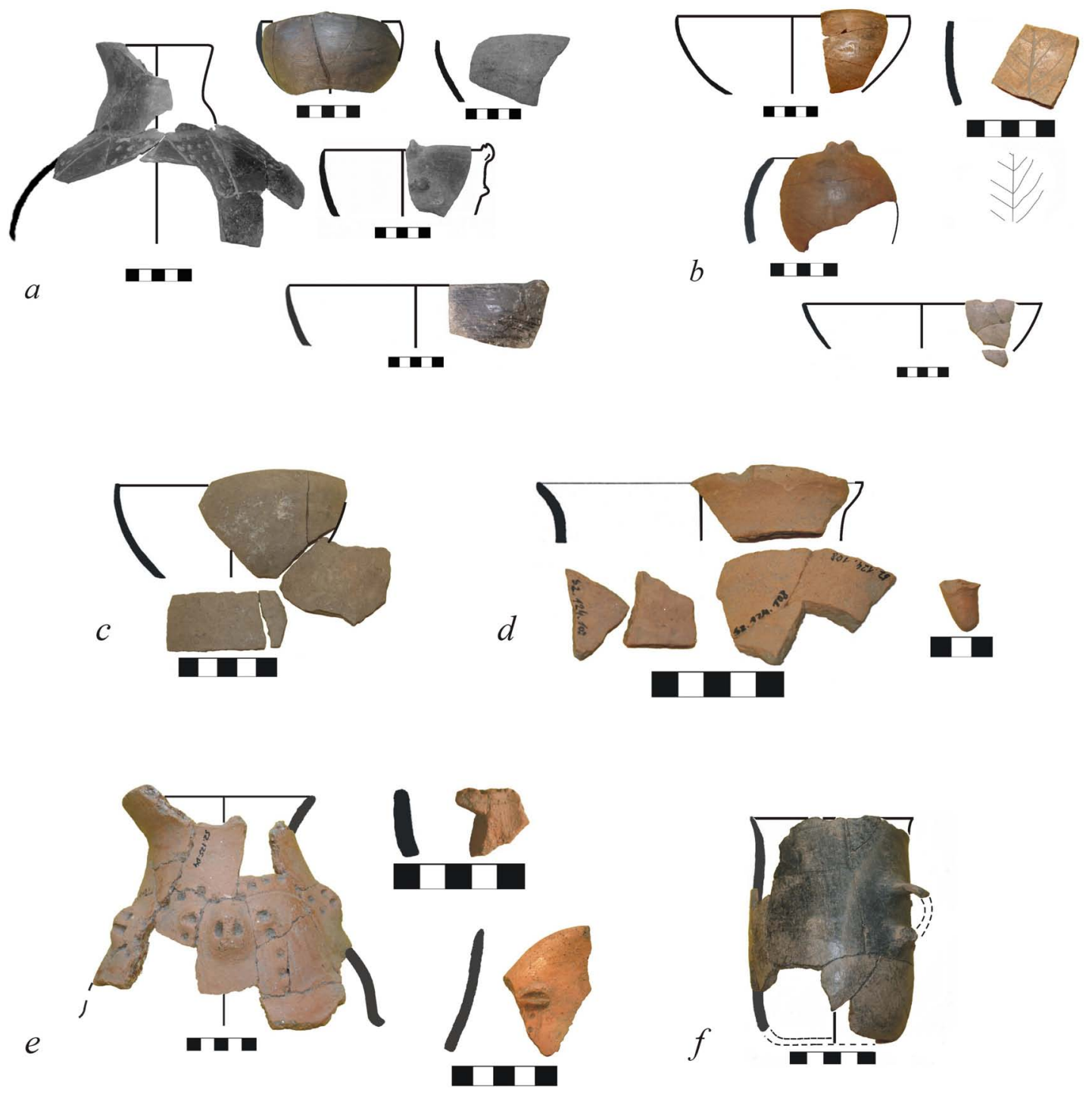

Figura 13. Algunos ejemplares de las modalidades estilísticas propuestas. $a$ : alfarerías plomizas pulidas; $b$ : alfarerías café pulidas; $c$ : alfarerías alisadas; $d$ : alfarerías naranja pulidas de pasta fina; $e$ : alfarerías naranja pulidas de pasta mediana; $f$ : vaso de estilo Tebenquiche.

Figure 13. Some examples of the proposed stylistic modes: a: leaden polished potteries; $b$ : polished brown pottery, c: smoothed pottery d: thin paste polished orange pottery; e: medium paste polished orange potteries; f: Tebenquiche style vessel.

un estimativo del $5 \%$ hasta un $80 \%$. Pertenecen a todas las categorías formales de contenedores, en su mayoría formas abiertas, y sobre todo, pucos no restringidos, tratados con distintas modalidades de pulido. Estas son las vasijas que mejor se ajustan a prácticas de servicio y consumo de comidas y bebidas (Orton et al. 1997; Rice 1987) y para mezclar y almacenar temporalmente alimentos (Bugliani 2008). Las bases identificadas como pertenecientes a estas categorías de formas fueron cóncavo-convexas, atributo que otorga estabilidad a los recipientes. El tratamiento de las superficies mediante pulido confiere impermeabilidad a las vasijas, favoreciendo la contención de sustancias líquidas. Se descartan prácticas como cocción de alimentos debido a que las formas empleadas habitualmente, según se puede apreciar merced a estudios actualísticos, responden a categorías como ollas y cántaros (Menacho 2001), y a que no se ha encontrado evidencia de termoalteración ni restos de hollín en ninguna de estas FF. De todos modos, no puede afirmarse que el servicio haya constituido el único uso específico para estas vasijas. Los vasos y botellones, por su parte, presentan condiciones óptimas para la contención de líquidos, y en el caso de los vasos, para su distribución individual (Bugliani 2008). La simetría dorsiventral de la FF 184, de modalidad estilística E, señala que la misma tuvo un cuello oblicuo, característica que permite verter líquidos. Resultó curiosa la presencia de formas denominadas cucharas o cucharones; los fragmentos hallados pertenecen a bordes de las partes cóncavas de estos objetos, en las cuales se contuvieron sustancias de manera transitoria.

Por otro lado, se empleó alfarería fina para cumplir un rol importante en eventos excepcionales significativos, 

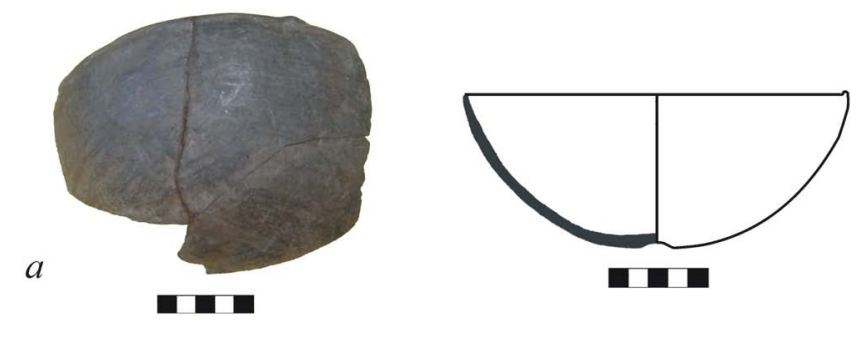

$b$
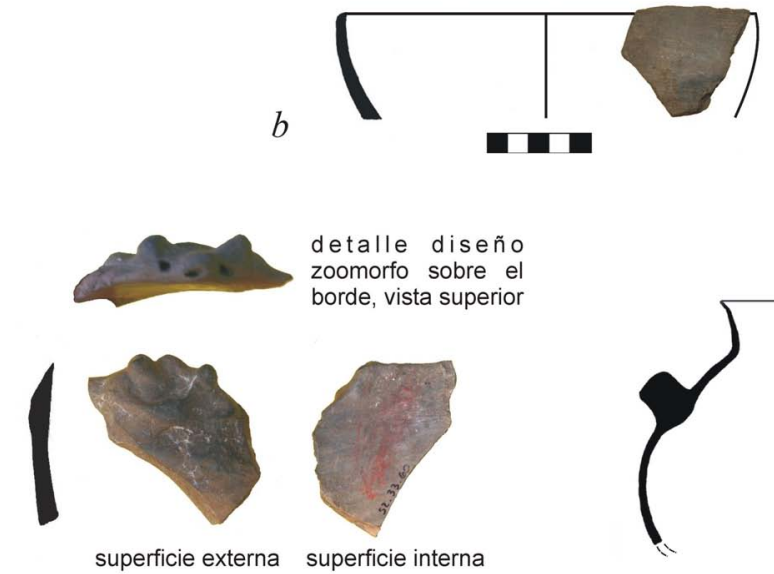

$c$

superficie externa superficie interna

a

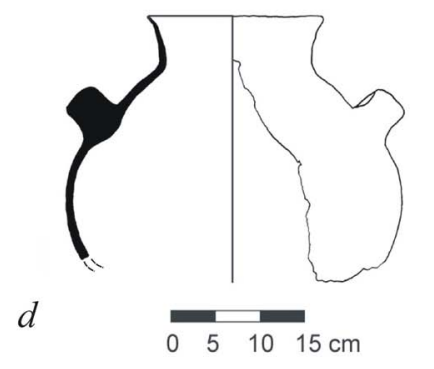

Figura 14. Alfarería vinculada al entierro 1. a: FF 217, puco de borde entrante y paredes convexas (izquierda: fragmentos remontados; derecha: reconstrucción gráfica de forma); $b$ : FF 224, puco no restringido de borde entrante y paredes convexas; c: FF 249, pequeño fragmento de puco no restringido de borde entrante $y$ paredes convexas, con modelado zoomorfo naturalista sobre el borde (vista del diseño modelado y de la aplicación de pintura roja post cocción sobre la superficie interna); d: olla globular ordinaria (esta última, extraída de Palamarczuk et al. 2007: 127, figura 5d).

Figure 14. Pottery associated with Burial 1: FF 217, bowl with incoming edge and convex walls (left: refitted fragments; right shape reconstruction); b: FF 224, unrestricted bowl with incoming edge and convex walls, with naturalistic zoomorphic modeling on the edge (view of the modeled design and of the application of post firing red paint on the inner surface); $d$ : ordinary globular pot (modified from Palamarczuk et al. 2007: 127, figure $5 d$ ).

que en términos cuantitativos las series ordinaria y fina se encuentran representadas en igual proporción. Esta relación cambia de considerarse las FF cuya asociación al entierro no es segura: siete FF $(87,5 \%)$ del conjunto fino contra una FF $(12,5 \%)$ del conjunto ordinario. como son las prácticas funerarias que tuvieron lugar luego del abandono de este espacio como área doméstica. Se trata de piezas vinculadas al entierro 1. La FF 217, correspondiente a medio puco no restringido de contorno simple de la modalidad A (Figura 14a), se usó como contenedor de huesos craneales de un subadulto. Media olla de tipo ordinario (Figura 14d) -la cual fue reutilizada para este uso funerario- cubría una diáfisis y dos porciones petromastoideas de subadulto ${ }^{10}$ y un instrumento de piedra (Spano et al. 2011). Junto a la olla, entre otros ítems, se encontraron seis fragmentos correspondientes a sendas FF dispuestos en posición horizontal; dado que se trata de una inhumación secundaria, existe cierta reserva respecto a la estricta asociación de estos fragmentos al entierro: al haberse echado tierra sobre la fosa para cubrirlo, cabe la posibilidad de que hayan sido incluidos de manera no intencional (Spano et al. 2011). Una FF corresponde a la modalidad A (\#224), otra a la modalidad B (\#249) y cuatro pertenecen al conjunto de alfarería fina sin asignación de modalidad estilística (\#222, \#223, \#227 y \#241). Se trató en todos los casos de pucos no restringidos de contorno simple; la única que permitió la reconstrucción gráfica de forma fue la FF 224 (Figura 14b); el resto corresponde a fragmentos muy pequeños de borde, el mayor de los cuales presenta un modelado zoomorfo (¿murciélago?) sobre el borde y pintura roja post cocción en la superficie interna (FF 249, Figura 14c). Si consideramos las vasijas certeramente asociadas al entierro -el medio puco \#217 y la mitad de olla- vemos

\footnotetext{
10 No pudo establecerse que estos elementos pertenecieran a un mismo individuo (Spano et al. 2011).
}

En la otra inhumación de este recinto, el entierro 3, se empleó una olla ordinaria como contenedor de un neonato, de características estilísticas similares a la media olla asociada al entierro 1 y a los fragmentos de ese grupo cerámico hallados en el piso de ocupación de la vivienda (Palamarczuk et al. 2007).

Como puede interpretarse a partir de la información contextual, las esferas de uso cotidiano y funerario no necesariamente se corresponden con un empleo exclusivo de alfarería ordinaria y fina, respectivamente: tanto en el piso de ocupación y sus rasgos asociados como en los entierros se emplearon ambas alfarerías, con predominio de vasijas de la serie ordinaria en ambos tipos de contexto. No se dio, tampoco, una presencia diferencial significativa de las distintas modalidades estilísticas planteadas para la serie fina, en relación a dichas esferas. En nuestro caso de estudio, entonces, no sería aplicable una dicotomía entre cerámica funeraria y doméstica: las personas hicieron uso de las vasijas en prácticas diferentes independientemente de las características de estilo de las mismas. Estas observaciones se sostienen también en lo que respecta al piso de ocupación y a la inhumación en el recinto 2 (Spano et al. 2011).

Por otra parte, a una escala espacial más amplia, la caracterización de modalidades estilísticas permitió observar una serie de elementos que exhiben afinidades con espacios circundantes: la profusión de pucos no restringidos grises y negros con pulido en líneas, el recurso de las técnicas de apliques al pastillaje para generar elementos de diseño no figurativos y biomorfos, los 
puntos incisos en representaciones de cejas y lágrimas, son recursos estilísticos que han sido reportados para los conjuntos finos de momentos formativos del valle de Yocavil y espacios cercanos como las Yungas, el área de Tafí del Valle, la falda occidental del Aconquija, el valle del Cajón, el valle Calchaquí y el oasis de Laguna Blanca (e. g. Bugliani 2008; Bugliani y Pereyra Domingorena 1999; Scattolin 2006). Este universo comparte a su vez modos de elaboración de objetos de lugares de otros ámbitos del NOA. La presencia de objetos de estilos Vaquerías y Tebenquiche podría aludir a la circulación a distancia de bienes y modos estilísticos, que desde momentos muy tempranos operó a nivel regional (Albeck 2000), vinculando e integrando comunidades aldeanas de distintas zonas.

\section{Consideraciones finales}

Esta caracterización representa una etapa en un proceso colectivo de investigación a largo plazo, en el cual se conjugan distintas líneas de evidencia, a los fines de lograr una comprensión mayor de la vida social en el pasado; la continuidad de los trabajos en laboratorio y en terreno en un sitio en el cual la cerámica se presenta en grandes cantidades, requiere de parámetros para un ordenado proceso de identificación de los hallazgos y para guiar el remontaje y la reconstrucción de formas. A nivel instrumental, entonces, se intentó proporcionar un corpus de información acerca de una parte del material cerámico de Soria 2.

La sistematización efectuada proporciona un panorama de la alfarería del sitio, la cual formó parte de la vida cotidiana de las personas y de situaciones especiales tales como los eventos de inhumación. La muestra que aquí presentamos no constituye un conjunto homogéneo; a través del análisis de atributos formales, tecnológicos y decorativos considerados conjuntamente, proponemos una clasificación posible de la variabilidad registrada. A nivel de la construcción de herramientas conceptuales para la comprensión de las prácticas acaecidas en el pasado, esta clasificación provee una primera aproximación a los modos de hacer vigentes en momentos de la ocupación del sitio. Esta sistematización puesta en juego con información contextual, permite acercarnos al rol de las vasijas en las prácticas llevadas a cabo en el recinto 1 , y también comenzar a indagar acerca de las posibles interrelaciones a distinta escala que vincularon a los ocupantes entre sí y con personas de otros ámbitos espaciales.

La continuidad en el estudio de esta cerámica posibilitará ajustar, reformular o desechar los planteos aquí esbozados, y contribuir a la construcción del conocimiento acerca del estilo de vida de las sociedades agropastoriles tempranas del sur de Yocavil.

Buenos Aires, 17 de octubre de 2011

\section{Agradecimientos}

Este artículo presenta una parte de la investigación de mi tesis de licenciatura, destinada al estudio del conjunto fino de la alfarería de Soria 2; agradezco a mi directora de tesis, Dra. Myriam Tarragó, por su orientación y acompañamiento durante el proceso, así como a todos los compañeros con los que compartimos años de campañas en Andalhuala. A Sol Grimoldi, Valeria Palamarczuk y Jenny Baigorria Di Scala por su asistencia técnica. A los evaluadores por sus observaciones y sugerencias; no obstante, lo expresado es de mi entera responsabilidad.

\section{Bibliografía}

Albeck, M. E. 2000. La vida agraria en los Andes del Sur. M. N. Tarragó (ed.), Nueva Historia Argentina: I. Los Pueblos Originarios y la Conquista, pp. 187-228, Sudamericana, Bs. As.

Álvarez Larrain, A., J. Baigorria Di Scala, C. Belotti, J. P. Carbonelli, S. Grimoldi, M. S. López; D. Magnifico, V. Palamarczuk, J. Ponce de León, R. Spano, G. Spengler, L. Stern Gelman y F. Weber. 2009. Avances en el estudio de un contexto doméstico formativo en el Valle de Yocavil. T. Bourlot, D. Bozzuto, C. Crespo, A. C. Hecht y N. Kuperszmit (eds.), Entre pasados y presentes II, Estudios Contemporáneos en Ciencias Antropológicas, pp. 369-382, Fundación de Historia Natural Félix de Azara - INAPL, Bs. As.

Aschero, C. 1988. Pinturas rupestres, actividades y recursos naturales: un encuadre arqueológico. H. Yacobaccio (ed.), Arqueología contemporánea argentina. Actualidad y perspectivas, pp. 109-143, Editorial Búsqueda, Bs. As.

Baigorria Di Scala, L. J. 2009. "El sitio Formativo Soria 2: Estudio Tecno Morfológico del conjunto cerámico ordinario". Facultad de Filosofía y Letras, UBA, Argentina. 117 páginas. Museo Etnográfico Juan B. Ambrosetti.

Baigorria Di Scala, J., R. C. Spano. 2007. Análisis preliminar de la cerámica del sitio formativo Soria 2 (Valle de Yocavil, Catamarca). Actas del XVI Congreso Nacional de Arqueología Argentina, Número Especial Revista Pacarina, tomo III, pp. 65-70, EdiUnJu, San Salvador de Jujuy.

Balfet, H., M. F. Fauvet-Berthelot y S. Monzón. 1992. Normas para la descripción de vasijas cerámicas. Centro de Estudios Mexicanos y Centroamericanos, México.

Belotti López de Medina, C. R. 2007. Consumo de camélidos en el Valle de Yocavil (pcia. de Catamarca) a inicios del Período Formativo, fines del primer milenio A.C.: zooarqueología del sitio Soria 2. Actas del XVI Congreso Nacional de Arqueología Argentina, Número Especial Revista Pacarina, tomo III, pp. 85-89. EdiUnJu, 
San Salvador de Jujuy.

Bourdieu, P. 1977. Outline of a Theory of Practice. Cambridge University Press, Cambridge.

Bugliani, M. F. 2008. Consumo y representación en el Formativo del sur de los Valles Calchaquíes (Noroeste argentino): Los conjuntos cerámicos de las aldeas del primer milenio A.D. B.A.R. International Series, S1174, J. y E. Hedges, Oxford.

Bugliani, M. F. 2010. Códigos estéticos, expresiones plásticas y modos de representación en la cerámica del Formativo en Yutopián (Valle del Cajón, Noroeste argentino). Revista del Museo de Antropología, 3: 21-32.

Bugliani, M. F., L. Pereyra Domingorena. 1999. Conjuntos cerámicos en el sitio formativo de "Bañado Viejo" (Tucumán). Actas del XIII Congreso Nacional de Arqueología Argentina, tomo II, pp. 347-358, UNC, Córdoba.

Cailleux, A. S/f. Notice sur le code des couleurs des sols. Boubée, Francia.

Conkey, M., C. Hastorf. 1990. The uses of style in archaeology. Cambridge University Press, Cambridge.

Earle, T. 1990. Style and iconography as legitimation in complex chiefdom. M. Conkey y C. Hastorf (eds.), The uses of style in archaeology, pp. 73-81, Cambridge University Press, Cambridge.

Garay de Fumagalli, M., M. B. Cremonte. 2002. Ocupaciones agropastoriles tempranas al sur de la quebrada de Humahuaca (Jujuy, Argentina). Chungara, 34 (1): 35-52.

Gell, A. 1998. Art and agency. An anthropological theory. Clarendon Press, Oxford.

González, A. R. 1960. Nuevas fechas de la cronología Arqueológica Argentina obtenidas por el método de Radiocarbón (IV). Resumen y Perspectivas. Revista del Instituto de Antropología, 1: 303-331.

Haber, A. F. 2006. Una arqueología de los oasis puneños. Domesticidad, interacción e identidad en Antofalla, primer y segundo milenios d.C. Universidad del Cauca \& Jorge Sarmiento, Córdoba.

Harris, E. C. 1991. Principios de estratigrafía arqueológica. Crítica, Barcelona.

Hegmon, M. 1992. Archaeological Research on Style. Annual Review of Anthropology, 21: 517-536.

Heredia, O. R. 1974. Investigaciones arqueológicas en el sector meridional de las Selvas Occidentales. Revista del Instituto de Antropología, 5: 73-132.

Heredia, O. R., J. A Pérez y A. R. González. 1974. Antigüedad de la cerámica polícroma en el Noroeste Argentino. Revista del Instituto de Antropología, 5: 133-151.

Hodder, I. 1990. Style as historical quality. M. Conkey y C. Hastorf (eds.), The uses of style in archaeology, pp. 44-51, Cambridge University Press, Cambridge.

Korstanje, M. A. 1998. Desempolvando antigüedades: consideraciones sobre el repertorio cerámico Vaquerías. Mundo de Antes, 1: 69-120.

Krapovickas, P. 1955. El yacimiento de Tebenquiche. Publicaciones del Instituto de Arqueología, Facultad de Filosofía y Letras, Universidad de Buenos Aires, Bs. As.

Levine, M. H. 1957. Prehistoric Art and Ideology. American Anthropologist, 59 (6): 949-964.

Menacho, K. 2001. Etnoarqueología de trayectorias de vida de vasijas cerámicas y modo de vida pastoril. Relaciones de la Sociedad Argentina de Antropología, 26: 119-144.

Miller, D. 1985. Artefacts as categories. A study of ceramic variability in Central India. Cambridge University Press, Cambridge.

Ortiz, G., A. Delgado. 2002. Estilo de variabilidad cerámica intrarregional. Un caso de estudio en la Quebrada de Humahuaca. Estudios Sociales del NOA, 5: 136-178.

Orton, C., P. Tyers y A. Vince. 1997. La cerámica en arqueología. Crítica, Barcelona.

Palamarczuk, V. 2002. "Análisis cerámico de sitios del bajo de Rincón Chico, Valle de Yocavil, Catamarca". Facultad de Filosofía y Letras, UBA, Argentina, 183 páginas. Biblioteca A. Cortazar (FFyL, UBA).

Palamarczuk, V., R. Spano, F. Weber, D. Magnifico, S. López y M. Manasiewicz. 2007. Soria 2. Apuntes sobre un sitio Formativo en el Valle de Yocavil (Catamarca, Argentina). Intersecciones en Antropología, 8: 121-134.

Rice, P. M. 1987. Pottery Analysis. A Sourcebook. The University of Chicago Press, Chicago.

Rosso, C., R. C. Spano. 2005-2006. Evidencias del uso de alucinógenos en pipas halladas en dos sitios tempranos de los Valles Calchaquíes. Arqueología, 13: 79-98.

Rouse, I. 1960. The Classification of Artifacts in 
Archaeology. American Antiquity, 25 (3): 313-323.

Rye, O. 1981. Pottery Technology: principles and reconstruction. Manuals on Archaeology 4, Taraxacum, Washington.

Scattolin, M. C. 2006. Contornos y confines del universo iconográfico precalchaquí del valle de Santa María. Estudios Atacameños, 32: 119-139.

Scattolin, M. C. 2007. Santa María antes del año mil. Fechas y materiales para una historia cultural. V. I. Williams, B. N. Ventura, A. B. M. Callegari y H. D. Yacobaccio (eds.), Sociedades Precolombinas Surandinas. Temporalidad, Interacción y Dinámica cultural del NOA en el ámbito de los Andes Centro-Sur, pp. 203-219, Instituto de Arqueología, Facultad de Filosofía y Letras, UBA, Bs. As.

Scattolin, M. C., M. F. Bugliani. 2005. Un repertorio surtido: las vasijas de los oasis de Laguna Blanca, Puna Argentina. Revista Española de Antropología Americana, 35: $51-74$

Shepard, A. 1957. Ceramics for the Archaeologist.
Publications 609, Carnegie Institution of Washington, Washington.

Spano, R. C. 2008. "Indagaciones sobre las sociedades aldeanas del Valle de Yocavil; análisis de la alfarería fina del sitio Soria 2 (Andalhuala, pcia. de Catamarca)". Facultad de Filosofía y Letras, UBA, 182 páginas. Museo Etnográfico Juan B. Ambrosetti.

Spano, R. C., M. S. Grimoldi y V. Palamarczuk. 2011. "Morir temprano. Entierros de infantes en un espacio doméstico formativo de Yocavil, Noroeste Argentino". Ms.

Tarragó, M. N. 1989. “Contribución al conocimiento arqueológico de las poblaciones de los oasis de San Pedro de Atacama, en relación con los otros pueblos puneños, en especial el sector septentrional del valle Calchaquí". Facultad de Humanidades y Artes, Universidad Nacional de Rosario, 625 páginas. Museo Etnográfico Juan $B$. Ambrosetti.

Tarragó, M. N. 1999. El Formativo y el surgimiento de la complejidad social en el Noroeste Argentino. P. Ledergerber-Crespo (ed.), Formativo Sudamericano, una Reevaluación, pp. 302-313. Abya-Yala, Quito. 